\title{
Relações entre Desenvolvimento da Gratidão e Tipos de Valores em Jovens
}

\author{
Lia Beatriz, de Lucca Freitas - Universidade Federal do Rio Grande do Sul, Porto Alegre, Brasil \\ Jonathan Richard Henry Tudge - University of North Carolina at Greensboro, Greensboro, United States \\ Fernanda Palhares - Universidade Federal do Rio Grande do Sul, Porto Alegre, Brasil \\ Andressa Carvalho Prestes - Universidade Federal do Rio Grande do Sul, Porto Alegre, Brasil
}

\begin{abstract}
Resumo
Investigam-se relações entre o desenvolvimento da gratidão e tipos de valores. Trezentos e noventa e seis jovens de 7 a 14 anos responderam a Wishes and Gratitude Survey. Previu-se que, com o aumento da idade, haveria (a) uma diminuição de valores hedonistas; (b) uma diminuição de gratidão concreta e um aumento de gratidão conectiva. A terceira hipótese foi a de que seria mais provável que jovens com valores hedonistas expressassem gratidão concreta e aqueles com valores relacionados ao bem-estar de outros (BEO) expressassem gratidão conectiva. Para testar essas hipóteses, utilizou-se análise de regressão (estimativa de curva e regressão logística). Com o aumento da idade, os valores hedonistas e a gratidão concreta diminuíram; não se verificou aumento de gratidão conectiva. Valores hedonistas relacionaram-se positivamente à gratidão concreta. Todavia, valores $\mathrm{BEO}$ não se associaram à gratidão conectiva.

Palavras-chave: gratidão, valores, desenvolvimento moral, crianças, adolescentes
\end{abstract}

The Relations between the Development of Gratitude and Types of Values in Youth

\begin{abstract}
We studied the relations between the development of gratitude and types of values. A total of 396 7- to 14-year-old youth group responded to the $W$ ishes and Gratitude Survey. We expected that, with the increase in age, there would be (a) a decrease of hedonistic values and (b) a decrease of concrete gratitude and an increase in connective gratitude. Our third hypothesis was that it would be more likely for the young with hedonistic values express concrete gratitude and those with values related to the well-being of others (WBO) to express connective gratitude. To test these hypotheses we used regression analysis (curve estimation and logistic regression). With an increase in age, hedonistic values and concrete gratitude declined; there was no increase in connective gratitude. Hedonistic values were related positively to concrete gratitude. However WBO values were not related to connective gratitude.

Keywords: gratitude, values, moral development, children, adolescents
\end{abstract}

Relaciones entre el Desarrollo de Gratitud y Tipos de Valores en Jóvenes

\begin{abstract}
Resumen
Se investigan las relaciones entre el desarrollo de gratitud y tipos de valores. Respondieron a la encuesta Wishes and Gratitude Survey 396 jóvenes de edades entre 7 y 14 años. Fue previsto que con el aumento de edad habría, (a) disminución de los valores hedonistas; (b) disminución de gratitud concreta y aumento de gratitud conectiva. La tercera hipótesis fue que sería más probable que jóvenes con valores hedonistas expresaran gratitud concreta y aquellos con valores relacionados al bienestar de otros (BEO) expresaran gratitud conectiva. Para testar estas hipótesis, se utilizó el análisis de regresión (estimación de curva y regresión logística). Con el aumento de edad, los valores hedonistas y la gratitud concreta disminuyen; no se verificó aumento de gratitud conectiva. Valores hedonistas se relacionaron positivamente con gratitud concreta. Aún los valores BEO no se asociaron a gratitud conectiva.

Palabras clave: gratitud, valores, desarrollo moral, niños, adolescentes
\end{abstract}

No decurso do desenvolvimento, crianças e adolescentes assimilam os valores de sua cultura e aprendem a hierarquizá-los (Freitas, 2003). Em nossa sociedade, tem-se dado grande importância ao ganho imediato (hedonismo), aos bens materiais (materialismo) e ao bem-estar individual (individualismo) ( $\mathrm{La}$ Taille \& Menin, 2009; O'Dougherty, 2002). Todavia, resultados de pesquisa sugerem que esses valores trazem prejuízos à qualidade de vida dos jovens. Por exemplo, adolescentes mais materialistas têm pior desempenho escolar, são mais ansiosos e infelizes
(Kasser, 2005) e menos motivados a contribuir com a sociedade (Froh, Bono, \& Emmons, 2010) que seus pares. Não há como nem por que educar os jovens de forma imune aos valores preponderantes na cultura contemporânea (Ferry, 2013). Cabe investigar, porém, como é possível contrapesar esses valores, que podem ser perniciosos à vida dos jovens.

Em estudos realizados com adultos, verificou-se uma relação negativa entre níveis de gratidão e de materialismo (e.g., Lambert, Fincham, Stilman, \& Dean, 2009; Polak \& McCullough, 2006). Encontraram-se 
resultados similares com adolescentes entre 14 e 19 anos de idade (Froh, Emmons, Card, Bono, \& Wilson, 2011). Esses achados sugerem que a gratidão seria uma forma de contrabalançar o materialismo. Faz sentido pensar também que a gratidão pode contribuir para a redução do hedonismo, visto que requer a conservação de uma satisfação no tempo (e.g., Bonnie \& de Waal, 2004; Godbout, 1997). Além disso, pode ser um antídoto ao individualismo, na medida em que implica uma valorização não apenas do benefício recebido, mas também do benfeitor (e.g., McConnell, 1993; Piaget, 1965/1977), dando visibilidade ao outro.

Os resultados desses estudos merecem atenção, pois sugerem um caminho possível para uma educação em valores capaz de forjar uma melhor qualidade de vida. Dessa forma, vale a pena investir em estudos que examinem relações entre valores. Conforme enfatizam duas diferentes teorias (e.g., Gouveia, Milfont, \& Guerra, 2014; Schwartz, 2012), os valores formam um contínuo e não um conjunto de motivações discretas. Isso significa que todo o sistema de valores se relaciona com qualquer outra variável de uma forma integrada. No entanto, quando se trata de crianças e adolescentes, é preciso considerar que são indivíduos em formação: aquilo que os jovens valorizam varia em função da idade, e há também evidências de que existe um desenvolvimento da gratidão (e.g., Baumgarten-Tramer, 1938; Freitas, Pieta, \& Tudge, 2011; Nelson et al., 2013; Tudge, Vadehra, Wang, Stone, \& Freitas, 2011; Rava \& Freitas, 2013). Assim, é importante que se considerem especificamente o desenvolvimento dos sistemas de valores e da gratidão durante a infância e a adolescência.

\section{Sistemas de Valores na Infância e Adolescência}

Toda conduta humana visa a um fim, o qual tem valor porque desejado. Uma forma, portanto, de se ter acesso àquilo que uma pessoa valoriza é investigar-se o que ela deseja. Neste artigo, define-se valor como um investimento afetivo que move a ação em determinada direção (Freitas, 2003; La Taille, 2006; Menin, Tavares, \& Moro, 2013; Piaget, 1954).

Mesmo estando presentes desde o início da vida, os sistemas de valores de cada pessoa constroem-se paulatinamente. Os primeiros valores são lábeis, mas a partir do momento em que a criança é capaz de coordenar meios e fins (por volta dos oito meses de idade, em média), os valores já começam a se subordinar uns (meios) aos outros (fins), tendo início a formação de sistemas. Quando a criança adquire a função simbólica (Delval, 2013), o valor de um objeto torna-se mais durável no tempo: a satisfação suscitada por um objeto ou pessoa não se limita apenas ao momento imediato. A criança é agora capaz tanto de lembrar quanto de postergar uma satisfação. Assim, pouco a pouco, além dos valores hedonistas, que se restringem ao ganho imediato, somam-se os valores virtuais (Piaget, 1954; 1965/1977), os quais têm uma durabilidade no tempo.

A assimilação pela criança dos valores de seu grupo cultural é mediada pelos adultos significativos para ela, notadamente, seus pais. Alguns pesquisadores encontraram, por exemplo, uma relação entre os níveis de materialismo das crianças e de seus pais (e.g., Carslon \& Grossbart, 1988; Goldberg, Gorn, Peracchio, \& Bammossy, 2003), o mesmo ocorrendo com os níveis de hedonismo (Knafo \& Schwartz, 2009). É graças também à interação com os adultos que a criança aprende que há valores que dizem respeito às ações humanas intencionais (Menin \& Tavares, 2013), os quais são denominados valores morais. Diversos estudos evidenciaram o impacto da socialização dos pais no desenvolvimento moral de seus filhos (e.g., Grusec, 2006; Thompson \& Winer, 2014; Wainryb \& Recchia, 2014; White \& Matawie, 2004). Os próprios jovens reconhecem a influência de seus pais em seus valores. Em uma pesquisa com estudantes do ensino médio, $67,60 \%$ dos participantes consideraram que seus pais tinham muita influência em seus valores (La Taille, 2006). Além da relação com os adultos, as interações da criança com seus pares são importantes para a construção dos sistemas de valores. Por exemplo, Bannerjee e Dittmar (2008) encontraram uma relação positiva entre os níveis de materialismo de crianças e de seus pares. Os amigos, porém, parecem ser menos influentes que os pais (La Taille, 2006).

Assim como a capacidade de operar liberta a criança e o adolescente das ilusões perceptivas, a vontade não os deixa à mercê da busca da satisfação de desejos imediatos, tornando o jovem capaz de definir quais são os fins prioritários para sua ação, ou seja, possibilita a hierarquização de valores e a construção de projetos de vida (Freitas, 2003; Damon, 2009). Com o início da adolescência, o jovem torna-se capaz de raciocinar sobre hipóteses e de refletir sobre valores (Piaget \& Inhelder, 1955/1970). Os valores predominantes na cultura não mais aparecem como os únicos corretos e possíveis; o adolescente questiona os valores aprendidos (e.g., Barni, Ranieri, Scabini, \& Rosnati, 2011; Vale \& Alencar, 2012). Além disso, às relações interpessoais, somam-se os vínculos com grupos sociais mais amplos, tais como grupos políticos e religiosos. Assim, 
o adolescente entra em contato com valores ideais, tornando ainda mais complexos os seus sistemas de valores. Em um estudo que enfocou o desenvolvimento de quatro valores (obediência, cooperação, responsabilidade e criatividade) em jovens entre 5 e 14 anos de idade, verificou-se um crescente nível de descentração na forma como os participantes definiram esses valores (Andrade, Camino, \& Dias, 2008). As definições do tipo impessoal, que inclui sentimentos idealistas, mutualidade nas relações sociais e uma visão sistêmica, apareceram com maior frequência nos adolescentes. Encontraram-se resultados similares em uma pesquisa sobre a adesão ao valor justiça: os adolescentes (14 a 17 anos) escolheram mais respostas com maior nível de descentração, isto é, baseadas em princípios universalizáveis e contratos firmados democraticamente de acordo com procedimentos justos, que as crianças (10 a 13 anos) (Menin, Bataglia, \& Moro 2013).

Os valores ideais (por exemplo, a justiça, a generosidade, etc.) são muitas vezes encarnados por pessoas (Comte-Sponville, 2007; Piaget \& Inhelder, $1955 / 1970)$, as quais são admiradas por suas qualidades de caráter ou virtudes morais. Esse é também o caso da gratidão, a qual é considerada uma virtude moral na maioria das culturas (McCullough, Kilpatrick, Emmons, \& Larson, 2001). A gratidão, contudo, não é inata; há evidências de que a gratidão se desenvolve durante a infância e a adolescência.

\section{Desenvolvimento da Gratidão em Crianças e Adolescentes}

A gratidão pode emergir quando uma pessoa - o benfeitor - presta um favor ou ajuda a outra - o beneficiário, o qual sente, por sua vez, que deve retribuir ao benfeitor prévio (e.g., Bonnie \& de Waal, 2004; Comte-Sponville, 2007; McConnell, 1993; Piaget, 1965/1977) se for possível e quando surgir uma oportunidade. Diversos estudos indicam que a gratidão está presente na vida de crianças e adolescentes (e.g., Baumgarten-Tramer, 1938; Nelson et al., 2013; Paludo, 2014; Rava \& Freitas, 2013).

No que diz respeito especificamente ao desenvolvimento da gratidão, destaca-se o estudo de Baumgarten-Tramer (1938), no qual mais de 1.000 crianças e adolescentes da cidade de Berna, na Suíça, responderam a duas perguntas: (a) $\mathrm{O}$ que tu mais queres? e (b) O que tu farias para a pessoa que te desse o que tu mais queres? As respostas dos jovens à segunda questão foram categorizadas em quatro formas de gratidão. A gratidão verbal (por exemplo, dizer "obrigado") foi encontrada com uma frequência semelhante em todas as idades (em média, entre 30\% e 48\% das respostas) e foi especialmente frequente nos jovens de 15 anos (72\%). A gratidão concreta (ou egocêntrica), na qual o beneficiário retribui com alguma coisa que tem valor para ele e não para o benfeitor, foi mais frequente nas respostas das crianças menores e decresceu com a idade. $\mathrm{Na}$ gratidão conectiva, o beneficiário retribui o favor com algo que vá ao encontro de um desejo ou necessidade do benfeitor; essa forma de gratidão ocorreu com maior frequência nas respostas das crianças acima de 11 anos. Segundo Baumgarten-Tramer (1938), essas diferenças devem-se ao fato de que as crianças mais velhas e os adolescentes, sendo menos egocêntricos, são mais capazes de considerar o ponto de vista do outro. Por fim, a gratidão finalística é aquela em que o beneficiário retribui o favor seja com uma ação que auxilia o objeto desejado seja com uma ação que promova seu desenvolvimento pessoal. Por exemplo, o jovem diz que seu maior desejo é ser jogador de futebol e que retribuiria ao seu benfeitor fazendo muitos gols. Essa forma de gratidão foi ilustrada com respostas de adolescentes de 14 anos. Os resultados desse estudo pioneiro são importantes, porque indicam que há diversas formas de gratidão, as quais são mais ou menos sofisticadas. Mesmo as crianças e os adolescentes utilizam outras formas para expressar gratidão, além das formas de polidez (Comte-Sponville, 2007) ensinadas pelos adultos.

Alguns pesquisadores dedicaram-se precisamente a examinar essas formas de polidez. Exemplo disso foi o trabalho de Gleason e Weintraub (1976), no qual se observou que apenas $21 \%$ das crianças com menos de 6 anos agradeceram a um adulto que lhes deu balas no dia de Halloween e mais de $80 \%$ das crianças acima de 10 anos agradeceram nessa mesma situação. Em um estudo sobre o juízo de crianças de 6, 9 e 12 anos referente à reação que o beneficiário deve ter frente a um ato generoso, Vale (2012) constatou, pelo contrário, que o emprego da fórmula verbal de agradecimento predominou entre as crianças de 6 anos ( $75 \%$ dos participantes) e diminuiu consideravelmente com o aumento da idade (30\% das respostas das crianças de 9 anos e 35\% daquelas de 12 anos).

A diferença entre polidez e gratidão foi considerada um desafio a ser enfrentado pelos pesquisadores em estudos com crianças (Bono \& Froh, 2009; Gordon, Musher-Eizenman, Holub, \& Dalrymple, 2004). O método de Baumgarten-Tramer (1938) trouxe resultados que contribuem para essa distinção. Assim, Freitas et al. (2011) realizaram um estudo com 430 jovens de 
7 a 14 anos de Porto Alegre - RS, utilizando as mesmas perguntas da pesquisadora suíça. Nessa amostra, verificou-se uma grande diminuição na frequência de gratidão concreta com o avanço da idade, e um aumento de gratidão conectiva na faixa dos 11 aos 14 anos de idade. Embora a frequência da gratidão verbal tenha aumentado um pouco com a idade, a relação entre grupo etário (7 a 10 anos e 11 a 14 anos) e essa forma de gratidão não foi estatisticamente significativa. A gratidão finalística apareceu em apenas duas respostas. Nesse estudo, não se encontraram diferenças significativas entre meninos e meninas. Esse é um aspecto que merece ser ainda investigado, visto que Gordon et al. (2004) constataram diferenças na forma como meninos e meninas experienciam a gratidão: os primeiros são mais gratos a objetos materiais e as segundas, às pessoas. No entanto, outros pesquisadores (e.g., Freitas, O’Brien, Nelson, \& Marcovitch, 2012; Paludo, 2008) não encontraram suporte empírico para a hipótese de diferenças entre os sexos.

Em um estudo realizado com 284 jovens norte-americanos entre 7 e 14 anos de idade (Tudge et al., 2011), analisaram-se também as respostas dos participantes à primeira pergunta inicialmente proposta por Baumgarten-Tramer (1938). Essas respostas foram categorizadas como valores: (a) hedonistas, (b) relativos ao bem-estar próprio (BEP) e (c) relacionados ao bem-estar de outros (BEO). Consideraram-se como valores hedonistas as respostas que expressavam a busca por um ganho imediato, tais como: a posse de objetos materiais, dinheiro, viagem de lazer ou turismo. Os valores relativos ao bem-estar próprio (BEP) encontraram-se em respostas que mencionaram benefícios pessoais a serem alcançados no futuro, por exemplo: viver em outro lugar visando melhorar sua vida, bem-estar psicológico, sucesso escolar, ambição profissional. Os valores relacionados ao bem-estar de outros (BEO) abarcaram aquelas respostas que continham benefícios para outros: membros da família, amigos ou a sociedade como um todo. Além disso, examinaram-se relações entre esses tipos de valores e as três principais formas de gratidão (verbal, concreta e conectiva). Os resultados da análise de estimativa de curva (uma subcategoria da regressão linear) indicaram que os valores hedonistas predominaram entre as crianças de 7 a 11 anos, enquanto os adolescentes (12 a 14 anos) apresentaram com maior frequência valores relacionados ao bem-estar próprio (BEP) e de outros (BEO). Quanto aos tipos de gratidão, os dados foram similares aos dos estudos anteriores: (a) a gratidão verbal não variou significativamente com a idade, (b) as crianças expressaram com maior frequência gratidão concreta e (c) os adolescentes, gratidão conectiva. No que tange às relações entre tipos de valores e formas de gratidão, constatou-se que os valores hedonistas predominaram entre os jovens que expressaram gratidão concreta, enquanto os valores relacionados ao bem-estar de outros (BEO) preponderaram entre aqueles que expressaram gratidão conectiva. Quanto aos valores relativos ao bem-estar próprio (BEP), não se encontrou uma relação estatisticamente significativa com nenhuma das formas de gratidão.

O estudo de Tudge et al. (2011) é importante, porque até onde se sabe foi a primeira tentativa de investigar sistematicamente as relações entre os tipos de valores e as formas de gratidão. Embora Baumgarten-Tramer (1938) tenha mencionado que não haveria um padrão consistente entre os valores dos jovens e as formas de gratidão, ela não apresentou uma análise sistemática das respostas a sua primeira questão.

\section{O Presente Estudo}

Neste artigo, apresenta-se um estudo que visa investigar relações entre o desenvolvimento da gratidão e tipos de valores. Quando se considera que investigar o que uma pessoa deseja é uma maneira de se ter acesso aos seus valores, entende-se que vale a pena: (a) analisar as respostas à primeira questão proposta por Baumgarten-Tramer (1938), ou seja, aquilo que as crianças e adolescentes de hoje mais valorizam e (b) examinar relações entre os tipos de valores dos jovens e as formas de gratidão.

É importante que se examinem relações entre o desenvolvimento da gratidão e tipos de valores, pois há evidências de que a gratidão poderia contrabalançar valores que têm sido prejudiciais à qualidade de vida das pessoas, como é o caso do exacerbado apego aos valores materiais (Froh et al., 2011; Lambert et al., 2009; Polak \& McCullough, 2006). No entanto, devem-se considerar os sistemas de valores e não apenas valores isolados (Gouveia et al., 2014; Schwartz, 2012). Esta pesquisa amplia o conhecimento sobre o desenvolvimento da gratidão, porque busca compreender essas relações à luz de uma teoria e de resultados de estudos recentes que abordam o desenvolvimento dos sistemas de valores na infância e adolescência. A pesquisa de Tudge et al. (2011) não abarcou esse aspecto. Além disso, os resultados até agora encontrados precisam ser confirmados e testados em outros contextos. Para que possa ser considerado como parte do desenvolvimento humano, é necessário que um fenômeno se encontre em 
contextos culturais diferentes e não apenas nos Estados Unidos da América (e.g., Flavell, 1982; Lourenço, 1992; Tudge \& Freitas, 2012).

Duas foram as principais perguntas que nortearam este estudo transversal: (a) há diferenças de idade relacionadas ao que as crianças e adolescentes mais valorizam e as formas como expressam gratidão? e, em caso afirmativo, (b) como as diferenças nos tipos de valores se relacionam às formas de expressar gratidão (concreta ou conectiva)? Uma primeira expectativa é que, com o aumento da idade, haveria uma diminuição da valorização do ganho imediato (hedonismo), uma vez que, pouco a pouco, os valores virtuais passam também a integrar os sistemas de valores dos jovens. Uma segunda hipótese, baseada em resultados de estudos anteriores (Baumgarten-Tramer, 1938; Freitas et al., 2011; Tudge et al., 2011) é a de que, com o aumento de idade, haveria uma diminuição na frequência de gratidão concreta e um aumento de gratidão conectiva. A relação entre gratidão verbal e idade é ainda uma questão de pesquisa em aberto, visto que alguns estudos sugerem que há um aumento de frequência com a idade (Gleason \& Weintraub, 1976) e outros não (Freitas et al., 2011; Tudge et al., 2011; Vale, 2012). No texto de Baumgarten-Tramer (1938), há certa ambiguidade quanto ao resultado encontrado em sua amostra: pode-se ler que, em cada idade, a gratidão verbal esteve presente entre $30 \%$ e $40 \%$ das respostas e em $72 \%$, no caso dos adolescentes de 15 anos. Não se sabe, porém, se houve ou não um aumento linear. A terceira hipótese refere-se à relação entre tipos de valores e formas de gratidão: é mais provável que os jovens com valores hedonistas expressem gratidão concreta e aqueles com valores relacionados ao bem-estar de outros (BEO) expressem gratidão conectiva.

\section{Método}

\section{Participantes}

A amostra foi composta por 396 crianças e adolescentes de 7 a 14 anos, os quais frequentavam três escolas da rede pública $(61,1 \%)$ e duas escolas da rede privada de Porto Alegre - RS, escolhidas por conveniência, isto é, de forma não aleatória. As meninas $(M=$ $136,80$ meses, $D P=24,10)$ compuseram $59 \%$ da amostra $(n=232)$ e os meninos $(M=130,10$ meses, $D P=$ $27,80) 41 \%(n=164)$. O poder da amostra ficou em $72,22 \%$. Excluíram-se nove participantes: (a) quatro, porque tinham 15 anos na ocasião da coleta de dados e (b) cinco, porque os dados (idade) estavam incompletos.

\section{Instrumento e Procedimentos Gerais}

Os participantes responderam por escrito a Wishes and Gratitude Survey - WAGS (Freitas, Tudge, \& McConnell, 2008), derivada de Baumgarten-Tramer (1938), composta por quatro questões, sendo as duas primeiras relevantes para este estudo: (a) $\mathrm{O}$ que tu mais queres? (b) O que tu farias para a pessoa que te desse o que tu mais queres? Realizou-se a coleta de dados em pequenos grupos, nas escolas, em uma sala indicada por cada instituição.

O projeto de pesquisa foi aprovado pelo Comitê de Ética em Pesquisa do Instituto de Psicologia da Universidade Federal do Rio Grande do Sul (protocolo $n^{\circ}$ 22485). A direção de cada instituição de ensino assinou um documento autorizando o estudo, e os pais ou responsáveis legais de todos os participantes assinaram um Termo de Consentimento Livre e Esclarecido.

\section{Procedimentos de Análise dos Dados}

Inicialmente, submeteram-se as respostas dos participantes a uma análise de conteúdo (Laville \& Dione, 1999), sendo que as respostas à primeira pergunta foram analisadas de acordo com as categorias propostas por Tudge et al. (2011) e as respostas à segunda questão conforme as categorias utilizadas em estudos anteriores (Baumgarten-Tramer, 1938; Freitas al., 2011; Tudge et al., 2011). Dois juízes codificaram independentemente as respostas: um juiz leu e classificou todas as respostas, e o outro, 25\% delas. Em função de que o número de participantes, em cada idade, não foi igual e de que alguns participantes deram mais de um tipo de resposta, analisou-se a proporção de respostas em cada idade. Para testar as hipóteses, utilizou-se análise de regressão (estimativa de curva e regressão logística binária), em SPSS 20.

\section{Resultados}

$\mathrm{Na}$ codificação das respostas, houve um elevado acordo entre os juízes. $\mathrm{O}$ índice de concordância interjuízes, estimado pelo coeficiente Kappa, variou entre 0,93 e 0,96 quanto aos tipos de valores (respostas à primeira questão da $W A G S$ ) e entre 0,70 e 0,98 no que diz respeito às formas de gratidão (respostas à segunda questão da $W A G S)$.

A primeira pergunta que norteou este estudo diz respeito a mudanças relacionadas à idade nos tipos de valores e nas formas de expressar gratidão de crianças e adolescentes. Na Tabela 1, apresenta-se a percentagem de jovens em cada idade que mencionaram valores 
hedonistas, relativos ao bem-estar próprio (BEP) e relacionados ao bem-estar de outros (BEO), bem como as percentagens relativas às principais formas de gratidão (verbal, concreta e conectiva). Os resultados das análises de estimativa de curva indicam que, com a idade, os valores hedonistas diminuíram significativamente $\left(R^{2}=0,52, p=0,04, b=-3,84\right)$, os valores relativos ao bem-estar próprio aumentaram significativamente $\left(R^{2}\right.$ $=0,81, p<0,01, b=7,27)$ e os valores relacionados ao bem-estar de outros também aumentaram $\left(R^{2}=0,49\right.$, $p=0,05, b=1,78)$. Assim, confirmou-se a expectativa sobre a diminuição dos valores hedonistas com o aumento da idade.

No que tange às formas de gratidão, a análise de estimativa de curva revelou que a expressão de gratidão concreta diminuiu com a idade $\left(R^{2}=0,74, p<0,01\right.$, $b=3,83$ ), conforme havia sido previsto. No entanto, não houve mudança em relação à gratidão conectiva $\left(R^{2}\right.$ $=0,003, p>0,89, b=0,16)$. Desta forma, a segunda hipótese foi apenas parcialmente confirmada, pois a expectativa de que, com o aumento da idade, haveria um aumento de gratidão conectiva não encontrou suporte empírico. Quanto à gratidão verbal, constatou-se um aumento significativo dessa forma de gratidão com a idade $\left(R^{2}=0,76, p<0,01, b=6,30\right)$.

A segunda questão de pesquisa refere-se à relação entre os tipos de valores e as formas de gratidão. Mais especificamente, formulou-se a hipótese de que seria mais provável que os jovens com valores hedonistas expressassem gratidão concreta e aqueles com valores relacionados ao bem-estar de outros (BEO) expressassem gratidão conectiva. Para testar as associações entre tipos de valores e formas de gratidão, utilizou-se regressão logística binária, com cada uma das três formas de gratidão como variável dependente dicotômica, e cada um dos três tipos de valores como variável independente. Controlou-se a idade (expressa em meses), uma vez que idade está claramente associada com tipos de valores e formas de gratidão.

O modelo de regressão logística foi significativo $\left(\chi^{2}[2]=18,44, p<0,001\right)$, explicou $6,40 \%$ da variância (Nagelkerke $\mathrm{R}^{2}$ ) e classificou corretamente $68,9 \%$ dos casos. Conforme havia sido previsto, valores hedonistas relacionaram-se significativamente à gratidão concreta $\left(B=0,59, S E=0,23\right.$ Wald $\chi^{2}[1]=6,31, \operatorname{Exp}(B)=$ $1,80, p=0,012)$ após controlar-se a idade. Participantes com valores hedonistas tiveram 1,80 vezes mais probabilidade de expressar gratidão concreta que aqueles que não mencionaram esse tipo de valor, após controlar-se a idade. No caso da relação entre valores BEO e gratidão conectiva, contudo, o modelo não foi significativo $\left(\chi^{2}[2]=3,34, p=0,19\right)$ e explicou apenas $1,20 \%$ da variância (Nagelkerke $\mathrm{R}^{2}$ ), embora tenha classificado corretamente $69,90 \%$ dos casos $(B=-0,58, S E=0,32$ Wald $\left.\chi^{2}[1]=3,55, \operatorname{Exp}(B)=1,79, p=0,068\right)$. Participantes com valores $\mathrm{BEO}$ tiveram 1,79 vezes mais probabilidade de expressar gratidão conectiva que aqueles que não citaram esse tipo de valor, após controlar-se a idade. O pequeno tamanho do efeito (indicado pelo Nagelkerke $\mathrm{R}^{2}$ ) deveu-se ao pequeno número de participantes que mencionou valores $\mathrm{BEO}$ e gratidão conectiva, com um poder de $49,75 \%$.

Não se levantou nenhuma hipótese específica sobre possíveis diferenças entre meninos e meninas, a partir da literatura. Todavia, uma vez que existe ainda certa controvérsia sobre o assunto, decidiu-se examinar

Tabela 1

Percentagens de Jovens por Idade que Escolheram os Tipos de Valores e as Formas de Gratidão

\begin{tabular}{lcccccccc}
\hline Idade em Anos & $\begin{array}{c}7 \\
(n=33)\end{array}$ & $\begin{array}{c}8 \\
(n=40)\end{array}$ & $\begin{array}{c}9 \\
(n=56)\end{array}$ & $\begin{array}{c}10 \\
(n=50)\end{array}$ & $\begin{array}{c}11 \\
(n=69)\end{array}$ & $\begin{array}{c}12 \\
(n=56)\end{array}$ & $\begin{array}{c}13 \\
(n=42)\end{array}$ & $\begin{array}{c}(n=50) \\
\text { Valores }\end{array}$ \\
$\quad$ & & & & & & & \\
$\quad$ Hedonistas & $81,80 *$ & 67,50 & 66,10 & 58,00 & 37,70 & 60,70 & 54,80 & 50,00 \\
$\quad$ BEP & 24,20 & 35,00 & 42,90 & 56,00 & 78,30 & 60,70 & 66,70 & 78,00 \\
$\quad$ BEO & 3,00 & 15,00 & 5,40 & 8,00 & 11,60 & 19,60 & 11,90 & 20,00 \\
\hline Gratidão & & & & & & & & \\
$\quad$ Verbal & 37,50 & 15,20 & 20,00 & 37,50 & 38,00 & 58,00 & 48,20 & 66,70 \\
$\quad$ Concreta & 20,80 & 48,50 & 40,00 & 42,90 & 28,00 & 23,20 & 28,60 & 16,70 \\
$\quad$ Conectiva & 58,30 & 30,30 & 32,50 & 23,20 & 40,00 & 23,20 & 33,90 & 21,40 \\
\hline
\end{tabular}

* As percentagens não totalizam 100 porque (a) algumas crianças escolheram mais de um tipo de valor ou expressaram mais de uma forma de gratidão e (b) algumas respostas não puderam ser codificadas em nenhuma dessas três formas de gratidão. 
se haveria alguma influência do sexo nos tipos de valores e nas formas de gratidão. Tendo em vista que as meninas $(M=136,80$ meses, $D P=24,10)$ eram significativamente mais velhas que os meninos $(M=130,10$ meses, $D P=27,80), F(1,394)=6,49, p=0,01$, e que os resultados indicaram que tanto os tipos de valores quanto as formas de gratidão estavam relacionadas à idade, incluiu-se idade em meses como covariável na análise de regressão logística binária para acessar a possível influência do sexo. Constatou-se que sexo não estava relacionado nem a valores hedonistas nem a valores relativos ao bem-estar próprio (BEP), mas estavam significativamente associados a valores $\mathrm{BEO}(B=$ $-0,90, S E=0,36$ Wald $\chi^{2}[1]=6,20, \operatorname{Exp}(B)=0,405$, $p=0,013)$ após controlar-se a idade, explicando $6,40 \%$ da variância. Especificamente, observou-se que $7 \%$ dos meninos e $16 \%$ das meninas escolheram valores relacionados ao bem-estar de outros (BEO). Verificou-se ainda que sexo não estava associado a nenhuma das três formas de gratidão.

\section{Discussão}

Neste artigo, examinaram-se relações entre a gratidão e tipos de valores em crianças e adolescentes, a partir de uma perspectiva evolutiva (Delval, 2013), tendo em vista que estudos anteriores indicaram que os sistemas de valores (e.g., Andrade et al., 2008; Vale \& Alencar, 2012; Menin, Bataglia, et al., 2013) e a gratidão (e.g., Baumgarten-Tramer, 1938; Freitas et al., 2012; Nelson et al., 2013; Rava \& Freitas, 2013) se desenvolvem desde a infância.

Em primeiro lugar, analisaram-se diferenças de idade relacionadas: (a) aos tipos de valores e (b) às formas de gratidão. Conforme previsto, verificou-se uma diminuição dos valores hedonistas com o aumento da idade. Além disso, constatou-se que os valores relativos ao bem-estar próprio (BEP) e relacionados ao bem-estar de outros (BEO) aumentaram. Esses resultados são congruentes com aqueles encontrados por Tudge et al. (2011) com jovens norte-americanos. Assim, ganha suporte empírico a ideia de que, paulatinamente, além dos valores hedonistas, os valores virtuais (Piaget, 1954; $1965 / 1977)$ passam a integrar os sistemas de valores dos jovens. Pode-se observar também um crescente nível de descentração nesse processo, conforme haviam indicado outros autores (Andrade et al., 2008; Menin, Bataglia, et al., 2013). Chama atenção, entretanto, que mesmo entre os adolescentes (12 a 14 anos): (a) a percentagem de valores hedonistas é ainda relativamente grande, variando entre $50 \%$ e $60,70 \%$ e (b) a proporção de valores relacionados ao bem-estar de outros (BEO) é pequena, variando entre $11,90 \%$ e $20 \%$ (ver Tabela 1 ). Esses resultados sugerem que os sistemas de valores dos jovens estão fortemente influenciados pelo hedonismo e o individualismo preponderantes na contemporaneidade (La Taille \& Menin, 2009; O’Dougherty, 2002).

Quanto às formas de gratidão, os resultados confirmaram apenas parcialmente a hipótese levantada, visto que, como em estudos anteriores (Baumgarten-Tramer, 1938; Freitas et al., 2011; Tudge et al., 2011), observou-se uma diminuição da gratidão concreta com a idade, mas não houve um aumento da gratidão conectiva. Esses resultados, contudo, são compatíveis com a ideia de que existem diversas formas de gratidão, as quais têm diferentes graus de complexidade. Na gratidão concreta, existe certa reciprocidade, visto que o beneficiário entende que deve retribuir ao seu benfeitor prévio; ele acredita, porém, que aquilo que ele valoriza é também valorizado por seu benfeitor. Trata-se de uma forma elementar de gratidão: é aquilo que se chama de recompensa. Diferentemente, a gratidão conectiva caracteriza-se por uma reciprocidade mais sofisticada, na medida em que o beneficiário considera que aquilo que para ele tem valor não é, necessariamente, valorizado pelo outro. Em outras palavras, a gratidão conectiva requer um maior nível de descentração que a gratidão concreta (Baumgarten-Tramer, 1938; Freitas et al. 2011; Piaget, 1954; Tudge et al., 2011). A gratidão requer a retribuição do bem com o bem: um ato generoso é retribuído com generosidade e, portanto, busca-se satisfazer uma necessidade ou um desejo singular do benfeitor (McConnell, 1993). Em síntese, quando se trata de desenvolvimento da gratidão, é importante considerar não apenas os níveis de gratidão, isto é, o quanto os jovens são gratos (e.g., Froh et al., 2014; Froh et al., 2011), mas também as diferentes formas de gratidão (Baumgarten-Tramer, 1938; Bonnie \& de Waal, 2004; Freitas et al., 2011; Piaget, 1954; Tudge et al., 2011).

Neste estudo, verificou-se ainda um aumento estatisticamente significativo de gratidão verbal com a idade. Essa forma de gratidão é utilizada, muitas vezes, como uma forma de polidez (Comte-Sponville, 2007) ensinada pelos adultos desde cedo às crianças quando recebem um presente ou algum tipo de ajuda. A gratidão verbal, contudo, pode ser usada para expressar um sentimento profundo de gratidão. Este seria o caso dos adolescentes, os quais podem ter dificuldade de falar a respeito de seus sentimentos e, por essa razão, 
recorreriam à gratidão verbal (Baumgarten-Tramer, 1938). Em síntese, subjacentes a uma mesma forma de gratidão, poderia haver processos psicológicos qualitativamente distintos. Nesse sentido, sugere-se a realização de uma pesquisa utilizando-se instrumentos que possibilitem acessar esses processos.

Pode-se pensar também que a forma como os jovens expressam gratidão é influenciada pela maneira como são educados. Os jovens aprendem com os adultos não apenas formas de polidez (Comte-Sponville, 2007), mas também outras formas de expressar gratidão. Dado o impacto da socialização dos pais no desenvolvimento moral de seus filhos (e.g., Grusec, 2006; Wainryb \& Recchia, 2014; Thompson \& Winer, 2014; White \& Matawie, 2004), faz sentido cogitar que pais com diferentes crenças e valores tenham maneiras distintas de educar os seus filhos sobre como demonstrar gratidão a um benfeitor prévio. Essa é outra questão que merece ainda ser investigada.

Em segundo lugar, examinaram-se relações entre os tipos de valores e as formas de gratidão. É importante que tenha sido confirmada a expectativa de que os jovens com valores hedonistas expressem mais provavelmente gratidão concreta, porque sugere a existência de uma relação entre o desenvolvimento da gratidão e os sistemas de valores dos jovens. Quando se trata de crianças e adolescentes, não basta se examinar apenas os níveis de gratidão (o quanto os jovens são gratos) (e.g., Froh, et al., 2014; Froh, Kashdan, Ozimkowski, \& Miller, 2009), pois existem diferentes formas de gratidão (Baumgarten-Tramer, 1938; Bonnie \& de Waal, 2004; Freitas et al., 2011; Piaget, 1954; Tudge et al., 2011) e cabe considerar-se os sistemas de valores (e.g., Gouveia et al., 2014; Schwartz, 2012), e não apenas um tipo de valor (Froh et al., 2011).

O fato de que não se tenha verificado uma relação estatisticamente significativa entre valores relacionados ao bem-estar de outros (BEO) e gratidão conectiva deve ser examinada com cautela. Cabe notar que relativamente poucos jovens mencionaram valores $\mathrm{BEO}$ e gratidão conectiva. Isso limitou o poder de encontrar resultados estatisticamente significativos. Além disso, a baixa frequência tanto desse tipo de valor quanto dessa forma de gratidão, nessa amostra, leva a cogitar se, de fato, não existiria uma relação entre eles, uma vez que ambos requerem um maior nível de descentração (Andrade et al., 2008; Baumgarten-Tramer, 1938; Freitas et al., 2011; Menin, Bataglia, et al., 2013; Piaget, 1954; Tudge et al., 2011). Neste sentido, vale a pena examinar essa possível relação em amostras maiores.
Quanto a diferenças entre os sexos, os dados encontrados são consistentes com aqueles de outros pesquisadores (Freitas et al., 2011; Freitas et al., 2012; Froh et al., 2009; Paludo, 2008), visto que não se verificaram diferenças significativas entre meninos e meninas no que diz respeito à gratidão. Esse é um aspecto que tem sido abordado na literatura sobre gratidão em jovens, desde que Gordon et al. (2004) encontraram diferenças na maneira como meninos e meninas vivenciam a gratidão. Os resultados indicaram, porém, uma diferença entre os sexos no que diz respeito aos valores BEO: as meninas mencionaram esse tipo de valor com maior frequência que os meninos. Esse dado é congruente com estudos que sugerem que as meninas dão maior importância que os meninos às relações interpessoais e a outras pessoas (e.g., Bromnick \& Swallow, 2001; Gordon et al., 2004). Pesquisas realizadas no Brasil sobre os sistemas de valores também têm evidenciado diferenças de gênero (e.g., Formiga, Santos, Viana, \& Andrade, 2008; Tamayo, 1988). Segundo Formiga et al., essas diferenças, provavelmente, devem-se aos papéis sociais atribuídos a homens e mulheres.

\section{Considerações Finais}

Até onde se sabe, este é o primeiro estudo a explicitar a importância de se examinar relações entre o desenvolvimento da gratidão - uma virtude moral (Comte-Sponville, 2007; McConnell, 1993; McCullough et al., 2001) - e diferentes tipos de valores em crianças e adolescentes (e.g., La Taille, 2006; Piaget, 1954; 1965/1977), não se limitando somente aos valores materiais (Froh et al. 2011).

É importante, porém, mencionar algumas limitações deste estudo. Em primeiro lugar, deve-se considerar o tamanho da amostra. Em futuras pesquisas, seria relevante examinar-se a associação entre valores relacionados ao bem-estar de outros (BEO) e gratidão conectiva em uma amostra maior. Neste estudo, a frequência desse tipo de valor foi relativamente pequena e pode ter sido responsável pela ausência de significância estatística. Por que valeria a pena se investigar novamente essa relação? Porque é a gratidão conectiva que cria ou fortalece os vínculos entre as pessoas (e.g., Baumgarten-Tramer, 1938; Bonnie \& de Waal, 2004; McCullough et al., 2001) e poderia servir de antídoto a valores que têm trazido prejuízos à qualidade de vida das pessoas. Em segundo lugar, constatou-se, mais uma vez, que existem diversas formas de gratidão, as quais têm diferentes níveis de complexidade. Resta compreender melhor: (a) 
os processos psicológicos subjacentes a essas formas de gratidão e (b) como essas formas de expressar gratidão são influenciadas pela educação em valores que os jovens recebem dos adultos. Nesse sentido, caberia conduzirem-se estudos sobre: (a) os processos psicológicos envolvidos no desenvolvimento da gratidão e (b) a influência dos valores dos pais nos tipos de valores e nas formas de gratidão dos filhos.

Apesar disso, considera-se que este estudo traz resultados relevantes e contribuiu para ampliar o conhecimento sobre o desenvolvimento da gratidão em crianças e adolescentes, na medida em que chama a atenção para a importância de se considerar os sistemas de valores de maneira integrada, ao invés de valores isolados. Além disso, os resultados encontrados sugerem caminhos para uma educação em valores que contribua para a promoção do bem-estar dos jovens.

\section{Referências}

Andrade, M. W. C. L., Camino, C., Dias, M. G. B. B. (2008). O desenvolvimento de valores humanos dos cinco aos 14 anos de idade: Um estudo exploratório. Revista Interamericana de Psicologia, 42(1), 19-27. Recuperado de http://pepsic.bvsalud.org/ pdf/rip/v42n1/v42n1a03.pdf

Bannerjee, R., \& Dittmar, H. (2008). Individual differences in children's materialism: The role of peer relations. Personality and Social Psychology Bulletin, 34(1), 17-31. doi: 10.1177/0146167207309196

Barni, D., Ranieri, S., Scabini, E., \& Rosnati, R. (2011). Value transmission in the family: Do adolescents accept the values their parents want to transmit? Jounal of Moral Education, 40(1), 105-121. doi: 10.1080/03057240.2011.553797

Baumgarten-Tramer, F. (1938). "Gratefulness" in children and young people. Journal of Genetic Psychology, 53, 53-66. doi: 10.1080/08856559.1938.10533797

Bonnie, K. E., \& de Wall, F. B. M. (2004). Primate social reciprocity and the origin of gratitude. Em R. A. Emmons \& M. E. McCullough (Eds.), The psychology of gratitude (pp. 213-229). New York: Oxford University Press.

Bono, G., \& Froh, J. J. (2009). Gratitude in school: Benefits to students and schools. In R. Gilman, E. S. Huebner \& M. Furlong (Eds.), Handbook of positive psychology in schools (pp. 77-88). New York: Routledge.
Bromnick, R. D., \& Swallow, B. L. (2001). Parties, lads, friends, love and Newcastle united: A study of young people's values. Educational Studies, 27, 143158. doi: $10.1080 / 03055690125235$

Carlson, L., \& Grossbart, S. (1988). Parental style and consumer socialization of children. Journal of Consumer Research, 15(1), 77-94. http://dx.doi. org/10.1086/209147

Comte-Sponville, A. (2007). Pequeno tratado das grandes virtudes (E. Brandão, Trad.). São Paulo: Martins Fontes.

Damon, W. (2009). O que o jovem quer da vida? Como pais e professores podem orientar e motivar os adolescentes. (J. Valpassos, Trans.) São Paulo: Summus.

Delval, J. (2013). O desenvolvimento psicológico bumano (R. A. Rosenbusch, Trad.). Petrópolis: Vozes.

Ferry, L. (2013). Do amor: Uma filosofia para o século XXI (R. Janowitzer, Trad.). Rio de Janeiro: Difel.

Flavell, J. H. (1982). Structures, stages, and sequences in cognitive development. In W. Collins (Ed.), The concept of development (pp. 1-28). Hillsdale, New Jersey: Lawrence Erlbaum.

Formiga, N. S., Santos, L. M. S., Viana, D. N. M., \& Andrade, A. C. (2008). Valores humanos e gênero. Psicologia \&m Foco, 1(1), 1-12. Recuperado de http://linux.alfamaweb.com.br/sgw/downloads/161_064132_3.pdf

Freitas, L. B. L. (2003). A moral na obra de Jean Piaget: Um projeto inacabado. São Paulo: Cortez.

Freitas, L. B. L., O’Brien, M., Nelson, J. A., Marcovitch, S. (2012). A compreensão da gratidão e teoria da mente em crianças de 5 anos. Psicologia: Reflexão e Crítica, 25(2), 330-338. doi: 10.1590/ S0102-79722012000200015

Freitas, L. B. L., Pieta, M. A. M., \& Tudge, J. R. H. (2011). Beyond politeness: The expression of gratitude in children and adolescents. Psicologia: Reflexão e Crítica, 24(4), 757-764. doi: 10.1590/ S0102-79722011000400016

Freitas, L. B. L., Tudge, J. R. H., \& McConnell, T. (2008). The wishes and gratitude survey. Greensboro, NC: Questionário não publicado (Adaptado e expandido a partir de Baumgarten-Tramer, 1938).

Froh, J. J., Bono, G., \& Emmons, R. (2010). Being grateful is beyond good manners: Gratitude and 
motivation to contribute to society among early adolescents. Motivation and Emotion, 34(2), 144-157. doi: 10.1007/s11031-010-9163-z

Froh, J. J., Bono, G., Fan, J., Emmons, R. A., Henderson, K., Harris, C., Leggio, H., \& Wood, A. M. (2014). Nice thinking! An educational intervention that teaches children to think gratefully. School Psychology Review, 43(2), 132-152. Recuperado de http://www.academia.edu/6647891/Nice_Thinking_An_Educational_Intervention_That_Teaches_Children_to_Think_Gratefully

Froh, J. J., Emmons, R. A., Card, N. A., Bono, G., \& Wilson, J. A. (2011). Gratitude and the reduced costs of materialism in adolescents. Journal of Happiness Studies, 12, 289-302. doi: 10.1007/ s10902-010-9195-9

Froh, J. J., Kashdan, T. B., Ozimkowski, K. M., \& Miller, N. (2009). Who benefits the most from a gratitude intervention in children and adolescents? Examining positive affect as a moderator. The Journal of Positive Psychology, 4, 408-422. doi: 10.1080/17439760902992464

Gleason, J. B., \& Weintraub, S. (1976). The acquisition of routines in child language. Language in Society, 5, 129-136. Recuperado de http://www.jstor.org/ stable $/ 4166866$ ?seq=1\#page_scan_tab_contents

Godbout, J. T. (1997). O espírito da dádiva (J. P. Cabrera, Trad.). Lisboa: Instituto Piaget.

Goldberg, M. E., Gorn, G. J., Peracchio, L. A., \& Bammossy, G. (2003). Understanding materialism among youth. Journal of Consumer Psychology, 13(3), 278-288. doi:10.1207/S15327663JCP1303_09

Gordon, A. K, Musher-Eizenman, D. R., Holub, S. C., \& Dalrymple, J. (2004). What are children thankful for? An archival analysis of gratitude before and after the attacks of September 11. Journal of Applied Developmental Psychology, 25(5), 541-553. doi: 10.1016/j.appdev.2004.08.004

Gouveia, V. V., Milfont, L. T., \& Guerra, V. M. (2014). Functional theory of human values: Testing its contente and structure. Personality and Individual Differences, 60, 41-47. doi: 10.1016/j. paid.2013.12.012

Grusec, J. E. (2006). The development of moral behavior and conscience from a socialization perspective. In M. Killen \& J. G. Smetana (Eds.), Handbook of moral development (pp. 243-265). Mahwah, NJ: Lawrence Erlbaum Associates.

Kasser, T. (2005). Frugality, generosity, and materialism in children and adolescents: In K. A. Moore \& L. H. Lippman (Eds.), What do children need to flourish? Conceptualizing and measuring indicators of positive development (pp. 357-373). New York: Kluwer/Plenum.

Knafo, A., \& Schwartz, S. (2009). Accounting for parent-child value congruence: Theoretical considerations and empirical evidence. In U. Schönpflug (Ed.), Cultural transmission: Psychological, developmental, social, and methodological aspects (pp. 269-295). New York: Cambridge University Press.

Lambert, N. M., Fincham, F. D., Stilman, T. F., Dean, L. R. (2009). More gratitude, less materialism: The mediating role of life satisfaction. The Journal of Positive Psychology, 4(1), 32-42. doi: 10.1080/17439760802216311

La Taille, Y. de (2006). Moral e ética: Dimensões intelectuais e afetivas. Porto Alegre: Artes Médicas.

La Taille, Y. de, \& Menin, M. S. S. (Eds.). (2009). Crise de valores ou valores em crise? Porto Alegre: Artmed.

Laville, C., \& Dionne, J. (1999). A construção do saber: Manual de metodologia da pesquisa em ciências humanas (L. M. Siman, Trad.). Porto Alegre: Artes Médicas.

Lourenço, O. M. (1992). Psicologia do desenvolvimento moral: teoria, dados e implicações. Coimbra: Almedina.

McConnell, T. (1993). Gratitude. Philadelphia: Temple University Press.

McCullough, M. E., Kilpatrick, S. D., Emmons, R. A., \& Larson, D. B. (2001). Is gratitude a moral affect? Psychological Bulletin, 127(2), 249-266. doi: 10.1037//0033-2909.127.2.249

Menin, M. S. S., Bataglia, P. U. R., \& Moro, A. (2013). Adesão ao valor justiça em crianças e adolescentes. Estudos em Avaliação Educacional, 24(56), 18-47. Recuperado de http://www.fcc.org.br/pesquisa/ publicacoes/eae/arquivos/1866/1866.pdf

Menin, M. S. S., \& Tavares, M. R. (2013). Por que eleger o tema sobre avaliação de valores. Estudos em Avaliação Educacional, 24(56), 12-17.

Menin, M. S. S., Tavares, M. R., \& Moro, A. (2013). Mensurando valores morais: Uma pesquisa com alunos do $6^{\circ}$ ao $9^{\circ}$ ano do ensino fundamental. Revista de Educação Pública, 22(49), 525-549. Recuperado de 
http://periodicoscientificos.ufmt.br/index.php/ educacaopublica/article/view/931

Nelson, J. A., Freitas, L. B. L., O’Brien, M., Calkins, S. D., Leerkes, E. M., \& Marcovitch, S. (2013). Preschool-aged children's understanding of gratitude: Relations with emotion and mental state knowledge. British Journal of Developmental Psychology, 31, 42-56. doi:10.1111/j.2044-835X.2012.02077.x

O'Dougherty, M. (2002). Consumption intensified: The politics of middle-class daily life in Brazil. Durham, NC: Duke University Press.

Paludo, S. S. (2008). Emocões morais e gratidão: Uma nova perspectiva sobre o desenvolvimento de jovens que vivem em situação de risco pessoal e social (Tese de doutorado não publicada). Programa de Pós-Graduação em Psicologia, Universidade Federal do Rio Grande do Sul, Porto Alegre - RS.

Paludo, S. S. (2014). As emoções no universo moral dos adolescentes. In L. F. Habigzang, Diniz, E. \& Koller, S. H. (Eds.), Trabalhando com adolescentes: Teoria e intervenção psicológica (pp. 164-179). Porto Alegre: Artmed.

Piaget, J. (1954). Les relations entre l'affectivité et l'intelligence. Paris: Sorbonne.

Piaget, J. (1977). Études sociologiques, $3^{\mathrm{a}}$ edição. Genève: Droz. (Texto original publicado em 1965).

Piaget, J., \& Inhelder, B. (1970). De la logique de l'enfant à la logique de l'adolescent, $3^{\mathrm{a}}$ edição. Paris: PUF (Texto original publicado em 1955).

Polak, E. L., \& McCullough, M. E. (2006). Is gratitude an alternative to materialism? Journal of Happiness Studies, 7, 343-360. doi: 10.1007/s10902-005-3649-5

Rava, P. G. S., \& Freitas, L. B. L. (2013). Gratidão e sentimento de obrigatoriedade na infância. Psico-USF, 18(3), 383-394. doi: 10.1590/ S1413-82712013000300005

Schwartz, S. H. (2012). An overview of the Schwartz Theory of Basic Values. Online Readings in Psychology and Culture, 2(1). doi: 10.9707/2307-0919.1116

Tamayo, A. (1988). Influência do sexo e da idade sobre o sistema de valores. Arquivos Brasileiros de Psicologia, 38(3), 91-104. Recuperado de http:// bibliotecadigital.fgv.br/ojs/index.php/abp/ article/view/21318

Thompson, R. A., \& Winer, A. C. (2014). Moral development, conversation, and the development of internal working models. In C. Wainryb \& H. E. Recchia (Eds.), Talking about right and wrong: Parent-child conversations as contexts for moral development (pp. 299-333). New York: Cambridge University Press. doi: 10.1017/CBO9781139207072

Tudge, J. R. H., \& Freitas, L. B. L. (2012). Internationalization, globalization and culture. Psicologia e Sociedade, 24(3), 547-556. doi: 10.1590/ S0102-71822012000300008

Tudge, J., Vadehra, P., Wang, Y., Stone, K., Freitas, L. (2011, março/abril). From materialism to social well-being: Links with gratitude among children and adolescents. Trabalho apresentado em 2011 Biennial Meetings of the Society for Research in Child Development, Montréal, Canadá.

Vale, L. G. (2012). Desenvolvimento moral: A generosidade relacionada à justiça e à gratidão sob a ótica das crianças (Tese de doutorado não publicada). Programa de Pós-Graduação em Psicologia, Universidade de São Paulo, São Paulo - SP.

Vale, L. G., \& Alencar, H. M. (2012). A generosidade em contraposição à obediência à autoridade: Juízos morais de crianças e adolescentes. In C. B. Rossetti \& A. C. Ortega (Eds.), Cognição, afetividade e moralidade (pp. 215-235). São Paulo: Casa do Psicólogo.

Wainryb, C., \& Recchia, H. E. (2014). Parent-child conversations as contexts for moral development: Why conversations, and why conversations with parents? In C. Wainryb \& H. E. Recchia (Eds.), Talking about right and wrong: Parent-child conversations as contexts for moral development (pp. 3-18). New York: Cambridge University Press. doi: 10.1017/ CBO9781139207072

White, F. A., \& Matawie, K. M. (2004). Parental morality and family processes as predictors of adolescent morality. Journal of Child and Family Studies, 13(2), 219233. doi: 10.1023/B:JCFS.0000015709.81116.ce

Recebido em: 25/11/2014

Primeira reformulação em: 17/03/2015

Aprovado em: 15/04/2015 
Nota dos autores:

Os autores agradecem o apoio financeiro do CNPq (Processo 301714/2012-6) e do Programa PIBIC UFRGS/CNPq. O segundo autor agradece ainda a CAPES pela bolsa de professor visitante sênior (Processo 18344-12-7).

Sobre os autores:

Lia Beatriz de Lucca Freitas é doutora em Psicologia pela Universidade de São Paulo (USP), professora do Programa de Pós-Graduação em Psicologia da Universidade Federal do Rio Grande do Sul (UFRGS), onde coordena o LAPEGE - Laboratório de Psicologia e Epistemologia Genética, bolsista de produtividade do CNPq e membro do grupo de pesquisa More than Thank You.

E-mail:1blf@ufrgs.br

Jonathan Richard Henry Tudge é Ph.D. em Psicologia do Desenvolvimento pela Cornell University (USA), professor titular na University of North Carolina at Greensboro (UNCG), onde coordena o grupo de pesquisa More than Thank You, e professor colaborador do Programa de Pós-Graduação em Psicologia da Universidade Federal do Rio Grande do Sul (UFRGS).

E-mail:jrtudge@uncg.edu

Fernanda Maria Palhares Castro é psicóloga e doutoranda do Programa de Pós-Graduação em Psicologia da Universidade Federal do Rio Grande do Sul (UFRGS), e membro do LAPEGE - Laboratório de Psicologia e Epistemologia Genética desde 2007.

E-mail: ferzinha.palhares@gmail.com

Andressa Carvalho Prestes é psicóloga pela Universidade Federal do Rio Grande do Sul, atuou como bolsista de Iniciação Científica (CNPq) de agosto de 2011 a julho de 2014 no LAPEGE - Laboratório de Psicologia e Epistemologia Genética, contribuindo em estudos com a temática dos conflitos em crianças pré-escolares e, mais recentemente, nos projetos a respeito da gratidão em crianças e adolescentes.

E-mail: andressa.prestes@ufrgs.br

Contato com os autores:

Lia Beatriz de Lucca Freitas

Programa de Pós-Graduação em Psicologia da UFRGS

Rua Ramiro Barcelos, 2600 - Bairro Santana

Porto Alegre - RS, Brasil

CEP: 90035-003

E-mail: lapege@lapege.com.br 